\title{
Considerations of pharmacogenetic testing in children
}

\author{
"With minimal risks of pharmacogenetic testing and potential \\ benefits, pharmacogenetic availability can help reduce pediatric \\ adverse events and treatment failure."
}

First draft submitted: 22 April 2016; Accepted for publication: 22 April 2016;

Published online: 1 June 2016

Keywords: benefits $\bullet$ children $\bullet$ pharmacogenetic testing $\bullet$ risks

Approximately $60 \%$ of US children currently take a prescribed medication [1]. Medication errors and biological differences in children increase the risk of adverse events (ADRs). Knowledge of pharmacogenetic (PGx) variants that impact ADR risk or drug efficacy may reduce these events by informing therapeutic decision-making and in turn reduce healthcare costs associated with suboptimal outcomes. Thus, the pediatric population may greatly benefit from pre-emptive PGx testing. We explore advantages and risks of pre-emptive PGx testing in newborns.

Drug response is a complex phenotype that warrants continued study to understand drug-drug, drug-gene and interactions with other clinical biomarkers. For example, recent work indicates that a child's PGx profile and mother's smoking habits contribute to response to methylphenidate for attention deficit hyperactivity disorder [2]. However, randomized clinical trials are unlikely to provide evidence of clinical utility for PGx; therefore, 'practical trials' may be an acceptable alternative, particularly for children and the elderly, who are under-represented in drug studies [3]. The impact of PGx variants for medications used in pediatric populations has been reviewed elsewhere [4], but includes codeine, atomoxetine, warfarin and voriconazole.

The advantages of providing PGx testing pre-emptively compared with the point-ofcare are unclear [5]. Several hospitals and clinics are exploring implementation of prospective PGx testing [6]. St. Jude Children's
Research Hospital (TN, USA) provides a PGx panel for all children admitted to their hospital, and Vanderbilt University tests all adult patients who consent to participate in their hospital-based biobank program for several PGx genes. The University of Maryland (MD, USA) and University of Florida (FL, USA) also offer PGx testing for adults undergoing catheterization.

\section{Pre-emptive PGx testing in children}

Current policies recommend that genetic testing of children only be considered if there is benefit to the child $[7,8]$. To respect autonomy, genetic testing of no benefit during childhood should be deferred until the child reaches legal age or attains maturity to assent. However, recent initiatives such as BabySeq (sequencing of healthy newborns) and professional recommendations to return secondary, disease-related genetic risk to all patients who undergo clinical sequencing [9] contradict these testing criteria.

Based on recommendations, PGx testing should only be ordered if useful during childhood. Imminent benefit or clinical utility of pre-emptive PGx testing has been exemplified by analyses indicating that over $90 \%$ of adult patients carry at least one PGx [10] and other groups have found that a substantial proportion of adult patients will be prescribed a medication with a known PGx interaction [11] and could benefit from pre-emptive testing.

While no similar analysis is yet available for pediatric populations, we find that 63 of

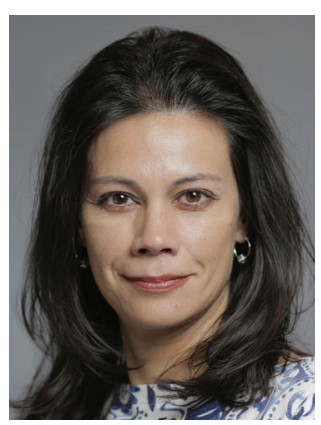

Susanne B Haga Department of Medicine, Center for Applied Genomics \& Precision Medicine, Duke University School of Medicine, 304 Research Drive, Box 90141, Durham, NC 27708, USA

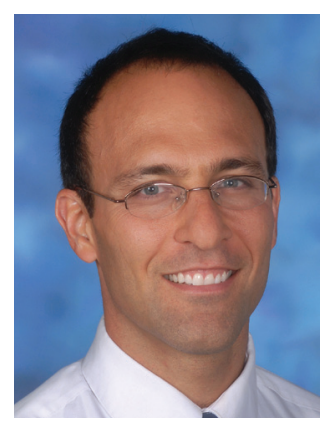

Benjamin D Solomon Author for correspondence: Division of Medical Genomics, Inova Translational Medicine Institute, Associate Professor, Virginia Commonwealth University School of Medicine, 3300 Gallows Road, 2nd Floor, Claude Moore Building, Falls Church, VA 22042, USA Tel.: +1 703776 6118/8199 Fax: +1 7037767177

benjamin.solomon@inova.org

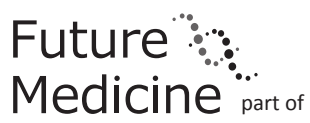


144 medications included in the US FDA's Table of Pharmacogenomic Biomarkers in Drug Labeling [12] are approved for pediatric uses. Sing et al. [13] identified eight drugs included in the British National Formulary for Children that are also listed in the FDA's Table of Pharmacogenomic Biomarkers in Drug Labeling. One of those drugs is codeine, a drug associated with several deaths in children found to be CYP2D 6 ultra-rapid or extensive metabolizers [14]. This triggered a black-box drug label warning advising against pediatric codeine use following tonsillectomy and/or adenoidectomy. Based on the prevalence of ultra-rapid and poor metabolizers in the USA, and the estimated number of patients prescribed codeine, more than 300,000 pediatric patients each year may be at risk for drug toxicity or ineffective treatment [15].

Furthermore, pre-emptive PGx may be particularly beneficial for acute care. In 2013, $18 \%$ of children (0-18 years) and $24 \%$ of children under the age of 6 years sought care at an emergency department at least once [16]. Pre-emptive PGx testing could prevent treatment delay in these cases.

\section{Risks \& limitations of pre-emptive PGx testing}

Risks and limitations of pre-emptive testing must be addressed to ensure benefit to pediatric patients. Like all genetic tests, PGx testing may have familial implications such as revealing non-paternity. However, family members may benefit from learning a child's PGx results or be motivated to seek testing themselves. For example, codeine is prescribed to about $10 \%$ of postpartum mothers for pain management [17] and knowledge of the newborn's and mother's CYP2D 6 status can inform treatment decisions.

Another risk to consider is the potential for psychosocial harms. Presumably, there will be no benefit and no harm in the absence of a drug exposure, similar to the evidence of minimal harms of carrier screening that only provides benefit if two carriers decide to have children. Some have suggested that PGx test-

\section{References}

1 Clavenna A, Bonati M. Drug prescriptions to outpatient children: a review of the literature. Eur. J. Clin. Pharmacol. 65(8), 749-755 (2009).

2 Pagerols M, Richarte V, Sanchez-Mora C et al. Pharmacogenetics of methylphenidate response and tolerability in attention-deficit/hyperactivity disorder. Pharmacogenomics J. doi:10.1038/tpj.2015.89 (2016) (Epub ahead of print).

3 Loo TT, Ross CJ, Sistonen J et al. Pharmacogenomics and active surveillance for serious adverse drug reactions in children. Pharmacogenomics 11(9), 1269-1285 (2010). ing raises fewer concerns than disease susceptibility testing [18].

\section{Informational needs for a newborn PGx program}

PGx testing at a young age will require educational resources and applications to promote result portability between providers and in transition to adult care. Parents have expressed interest in PGx testing but need educational resources to promote informed decision-making [19]. This will enable understanding of potential lifetime benefits and promote result sharing with providers. Resources such as PharmGKB, the FDA's PGx Table, and commercial laboratories will support widespread PGx use. Informed consent may also address informational needs and support understanding. Similarly, clinical decision tools are needed to facilitate the use of PGx results [20]. Clinical utility may be realized and increased portability of results may be achieved by integrating results from pre-emptive PGx testing into the EHR.

\section{Conclusion}

Children account for a substantial proportion of drug expenditures and related care. With minimal risks of PGx testing and potential benefits, PGx availability can help reduce pediatric ADRs and treatment failure. However, as the level of evidence has not reached a threshold for inclusion in routine newborn testing, the option for PGx testing should be discussed with parents, informational tools should be made available, and written consent should be obtained.

\section{Financial \& competing interests disclosure}

SB Haga is a paid consultant to Mako Medical Laboratories (NC, USA) and the Inova Translational Medicine Institute (VA, USA). The authors have no other relevant affiliations or financial involvement with any organization or entity with a financial interest in or financial conflict with the subject matter or materials discussed in the manuscript apart from those disclosed.

No writing assistance was utilized in the production of this manuscript.

4 Rieder MJ, Carleton B. Pharmacogenomics and adverse drug reactions in children. Front. Genet. 5, 78 (2014).

5 Schildcrout JS, Denny JC, Bowton E et al. Optimizing drug outcomes through pharmacogenetics: a case for preemptive genotyping. Clin. Pharmacol. Ther. 92(2), 235-242 (2012).

6 Dunnenberger HM, Crews KR, Hoffman JM et al. Preemptive clinical pharmacogenetics implementation: current programs in five US medical centers. Annu. Rev. Pharmacol. Toxicol. 55, 89-106 (2015).

7 Committee On B. Committee on Genetics and, American College of Medical Genetics and, Genomics S, Ethical, Legal Issues C. Ethical and policy issues in genetic testing and 
screening of children. Pediatrics 131(3), 620-622 (2013).

8

ritish Society for Human Genetics. Report on the genetic testing of children 2010.

https://securehost11.zen.co.uk/

9 Green RC, Berg JS, Grody WW et al. ACMG recommendations for reporting of incidental findings in clinical exome and genome sequencing. Genet. Med. 15(7), 565-574 (2013).

10 Van Driest SL, Shi Y, Bowton EA et al. Clinically actionable genotypes among 10,000 patients with preemptive pharmacogenomic testing. Clin. Pharmacol. Ther. 95(4), 423-431 (2014).

11 Carpenter JS, Rosenman MB, Knisely MR, Decker BS, Levy KD, Flockhart DA. Pharmacogenomically actionable medications in a safety net health care system. SAGE Open Med. 4, 2050312115624333 (2016).

12 FDA. Table of pharmacogenomic biomarkers in drug labels. www.fda.gov/

13 Sing CW, Cheung CL, Wong IC. Pharmacogenomics-how close/far are we to practising individualized medicine for children? Br. J. Clin. Pharmacol. 79(3), 419-428 (2015).

14 Kelly LE, Rieder M, Van Den Anker J et al. More codeine fatalities after tonsillectomy in North American children. Pediatrics 129(5), e1343-e1347 (2012).

15 Kaiser SV, Asteria-Penaloza R, Vittinghoff E, Rosenbluth G, Cabana MD, Bardach NS. National patterns of codeine prescriptions for children in the emergency department.
Pediatrics 133(5), e1139-e1147 (2014).

16 U.S. Department of Health and Human Services, Centers for Disease Control and Prevention, National Center for Health Statistics. Table 79. Emergency department visits within the past 12 months among children under age 18, by selected characteristics: United States, selected years 1997-2013. In: Health, United States, 2014 (Eds). www.cdc.gov

17 Smolina K, Weymann D, Morgan S, Ross C, Carleton B. Association between regulatory advisories and codeine prescribing to postpartum women. JAMA 313(18), 1861-1862 (2015).

18 Hoop JG, Lapid MI, Paulson RM, Roberts LW. Clinical and ethical considerations in pharmacogenetic testing: views of physicians in 3 "early adopting" departments of psychiatry. J. Clin. Psychiatry 71(6), 745-753 (2010).

19 Zhang SC, Bruce C, Hayden M, Rieder MJ. Public perceptions of pharmacogenetics. Pediatrics 133(5), e1258-e1267 (2014)

20 Devine EB, Lee CJ, Overby CL et al. Usability evaluation of pharmacogenomics clinical decision support aids and clinical knowledge resources in a computerized provider order entry system: a mixed methods approach. Int. J. Med. Inform. 83(7), 473-483 (2014). 